\title{
Analytical and Numerical Study of the Temperature Distribution for a Solid Sphere subjected to a Uniform Heat Generation
}

\author{
Hussein K. Jobair \\ University of Baghdad \\ College of Engineering \\ Energy Engineering Department
}

\begin{abstract}
The effect of the constant and variable thermal conductivity on the temperature distribution for different materials had been carried out. A uniform heat generation was supplied to each of the selected materials. Three materials were chosen for this study (Copper, Aluminum and Iron). Analytical solution with MATLAB programming had been accomplished for the equation of temperature distribution for a solid sphere with steady state conditions. ANSYS simulation had been executed to ensure the results numerically. The results show that the effect of thermal conductivity to the temperature distribution in the case of Iron model is very significant due to the low thermal conductivity of Iron. The curvature of temperature distribution for Iron model is higher humping than the other selected materials (Aluminum and copper). The comparison between the analytical and numerical gives a very good agreement with a percentage error almost non-existent.
\end{abstract}

\section{Keywords}

Solid sphere, thermal conductivity, temperature coefficient of thermal conductivity, temperature distribution.

\section{INTRODUCTION}

The study of the effect of temperatures variation to the value of thermal conductivity is an important topic that concerning many engineering applications. The most important materials that extremely affected by the variation of temperature and consequently the value of the thermal conductivity is Metals. Metals are having different behaviors for the temperature distribution with respect to the thermal conductivity variation. Some metals may be exposed to increasing in the value of the thermal conductivity when the temperature supplied is increases. On the other hand, other metals exposed to decreasing in the value of the thermal conductivity when the value of the temperature supplied increasing.

Tzong Lu and Sen Kou [1] studied experimentally and numerically the effect of the thermal conductivity of a porous material with spherical inclusions. The study had been carried out for a tetragonal and simple cubic array models. The experimental part of this study was measured the effective thermal conductivity. Numerical simulation with finite element method had been carried out to present a three dimensional heat conduction model with complex geometry. The comparison between the experimental and numerical results had been achieved and showed a good agreement with the other models from Rayleigh, Davis and Maxwell.

Unal [2] presented an analytical solution for a one dimensional steady state temperature distribution equation. The study had been carried out on a plate model. The assumption which was used in this study is linearly temperature dependent thermal conductivity with a uniform internal heat generation. The boundary conditions that had been used in this study were: the temperature in the base of plate is constant and that the plate is thermally insulated at this location. Legendre's normal elliptical integral for the first and second kind including in this study to solve the temperature distribution equation. PFANN [3] in his study determine the temperature distribution for several element types. Heat generation was assumed to be as an internal source of heat within the solid model. The thermal conductivity was assumed to be varying with the temperature in one dimension problem. The one dimensional problem was reduced to a double integral problem. The selected models for this study were: flat plate with two different thicknesses, cylindrical rod cooled by a liquid with a certain temperature, cylindrical rod with internal heat generation and spherical shell with internal heat source. Jobair [4] investigated the effect of the temperature variation to the value of the thermal conductivity. The study had been accomplished analytically with some assumptions. The assumptions that had been made for this study is that the thermal conductivity varies linearly with the temperature. Other assumption had been put it for this study was there is a uniform heat source inside the model. The model under study was a flat plate with two cases; the same temperature at both sides of the plate and the other case is different temperature values at both sides. Different types of materials have been used in this study to confirm and compare the results. Ganji et. al. [5] evaluated the temperature distribution of annular fin with temperature dependent thermal conductivity by using Homotopy perturbation method (HPM). This study had been used this method for solving a nonlinear heat transfer equation which is assumed that the thermal conductivity is variable. An approximation analytical solution had been carried out by method of (HPM) in the form of an infinite power series. The comparison between the study and other numerical method had been achieved. This comparison proved that (HPM) provides a highly accurate analytical solution in the case of nonlinear problems. Wei et. al. [6] investigated the effect of thermal conductivity for a composite sphere on the inclusion surface. The study assumed that the composite spherical particle embedded in a homogenous matrix. The behavior of thermal conductivity was assumed to be varying according to the exponential function $\mathrm{K} i \mathrm{i}=\mathrm{c}$ $\exp (\beta r)$ (where $\beta$ is the temperature coefficient of the thermal conductivity and $r$ is the radius of the sphere). The effective medium approximation (EMA) method was used to estimate the effective thermal conductivity. Bussolino et. al. [7] developed a new technique to measure the thermal conductivity. The experiment was executed on a specimen 
subjected to a high temperatures and current pulse. The temperatures profile was measured during the free cooling period. The advantages and disadvantages of complementary techniques were presented to evaluate the thermal conductivity as a function of temperature. Computer simulation had been carried out for generating temperature profiles numerically. The comparison was achieved and good agreement was obtained.

The objective of this work is to study the effect of temperature variation to the thermal conductivity for a solid sphere analytically and numerically. Temperature distribution for a constant and variable thermal conductivity will be compared by deriving Poison's equation. A uniform heat generation subjected to the model under study. Different selected types of materials have been chose to accomplish this study and to compare between these selected materials. The range of temperatures which used in this study having a different values beginning from $0^{\circ} \mathrm{C}$ passing through melting point temperature for each selected material.

\section{STUDY DESCRIPTION}

Several assumptions have been putted for beginning with this study. Three different metals (Copper, Aluminum and Iron) have been selected to ensure the analytical and numerical work presented. The model was a solid sphere subjected to a constant surface temperature with inside uniform heat generation available. Fig. 1 shows a profile of a solid sphere with a constant and uniform heat generated inside the sphere. The equation of temperature distribution of a sphere with heat generation which is called poison's equation can be represented by equation (1):

$\frac{1}{\mathrm{r}^{2}} \frac{\partial}{\partial \mathrm{r}}\left(\mathrm{kr}^{2} \frac{\partial \mathrm{T}}{\partial \mathrm{r}}\right)+\dot{\mathrm{q}}=0$

Where $r$ represented the radius of the solid sphere from the center, $\mathrm{k}$ is the thermal conductivity of the metal model and $\dot{\mathrm{q}}$ represented the uniform heat generation inside the sphere.

From this point, the study will be divided into two cases. The first case with the assumption of the thermal conductivity is constant. The second case assumed that the temperature dependent thermal conductivity. For both cases, the boundary conditions that have been applied to the equation (1) are:

at $\mathrm{r}=0, \frac{\partial \mathrm{T}}{\partial \mathrm{r}}=0 \quad$ at $\mathrm{r}=\mathrm{R}, \quad \mathrm{T}=\mathrm{T}_{\mathrm{S}}$

For the first case (constant thermal conductivity) and by applying the boundary conditions, the general solution of the equation (1) is:

$\mathrm{T}(\mathrm{r})=\mathrm{T}_{\mathrm{s}}+\frac{\dot{\mathrm{q}} \mathrm{R}^{2}}{6 \mathrm{k}}\left(1-\frac{\mathrm{r}^{2}}{\mathrm{R}^{2}}\right)$

Where $\mathrm{R}$ and $\mathrm{T}_{\mathrm{s}}$ are shown in the Fig. 1 which is the radius and the surface temperature of the sphere respectively.

In the case of variable thermal conductivity which is the second case in this study. Thermal conductivity assumed to be variable with temperature. There are several criteria that governing the relation between the thermal conductivity and the temperature. One of these is that to be assumed that the thermal conductivity changing linearly with the temperature. The relation for this criterion can be simply explained by the equation (3) [8]:

$\mathrm{k}=\mathrm{k}_{0}(1+\beta \mathrm{T})$

Where $k_{0}$ is the thermal conductivity at a reference temperature. In this study the reference temperature was took $0^{\circ} \mathrm{C}$. $\beta$ Represented a temperature coefficient of the thermal conductivity which is having a fixed and specific value for each material.

Equation (3) is very acceptable for many studies, which is adopted for this study.

By applying the same boundary conditions mentioned above. The general solution of the equation (1) is:

$\mathrm{T}(\mathrm{r})=-\frac{1}{\beta} \pm \sqrt{\left(\frac{1}{\beta}+\mathrm{T}_{\mathrm{S}}\right)^{2}+\frac{\dot{\mathrm{q}} \mathrm{R}^{2}}{3 \mathrm{k}_{0} \beta}\left(1-\frac{\mathrm{r}^{2}}{\mathrm{R}^{2}}\right)}$

The derivation of equation (2) and (4) in appendix-A

\section{RESULTS AND DISCUSSION}

Analytical and numerical results were presented to evaluate the temperature distribution of a solid sphere with a uniform heat generation inside. The comparison between two cases had been carried out. The first case is a constant thermal conductivity, while the second case dealing with variable thermal conductivity with the temperature supplied. The reason for using several metals for this study is to explain the effect of the thermal conductivity to the temperature distribution for each selected metal and to compare with each other.

Fig. 2 shows the temperature distribution in the case of a constant thermal conductivity. The effect of a uniform heat generation inside the solid sphere is identical for all three selected metals (Copper, Aluminum and Iron). The behavior of the temperature distribution for these three metals shows the same pattern. The difference between these metals is in the value of the maximum temperature reaches in each metal. Iron having the maximum temperature value which is located at the center of sphere $(r=0)$. These results due to the value of the thermal conductivity of Iron which is be the lowest value in a comparison with the other thermal conductivity values of selected metals in this study. The lowest value of thermal conductivity in Iron causes to the curve of temperature distribution appear more humping than the other

curve related to the other selected metals.

Fig. 3 illustrates the temperature distribution of a solid sphere in the case of the thermal conductivity is varying with the temperature. This figure shows that the behavior of the temperature distribution is similar to the behavior of the temperature distribution in the case of the thermal conductivity is constant. The difference between Fig. 2 and Fig. 3 is the value of maximum temperature of each selected metals for this study. This difference in the maximum temperature becomes important when the temperature supplied at the surface of the solid sphere is high. Iron kept the highest temperature in comparison with the other selected materials for this study. This is because the same reason in the case of the constant thermal conductivity which is the thermal conductivity of Iron is small in comparison with the other selected metals.

From Fig. 2 and Fig. 3, the variation of the values of the temperature depended upon the radial direction. This is means that the value of temperature is constant for any angle for a certain radius.

Numerical results were obtained from ANSYS by applying the same boundary conditions that had been chosen for the analytical results.

Fig. 4 and Fig. 5 show the results of Iron in the case of the constant and variable thermal conductivity. Because of the symmetry of the temperature distribution for a solid sphere, 
just a quarter of a solid sphere shown in these figures. Fig. 4 and Fig. 5 illustrate that the maximum temperature was found in the center of the solid sphere $(r=0)$. These figures also show the gradient in the temperature from the center of the solid sphere. The maximum value of temperature appears in the center of sphere. The gradient begins from the center of the sphere to the surface of the sphere which is having the minimum value of temperature.

The behavior of the temperature distribution for other metals under this study is the same as the behavior of Iron in Fig. 4 and Fig. 5. The difference between these models is that Iron having the maximum temperature value in the center in both cases (constant and variable thermal conductivity. The reason of that is the thermal conductivity. The thermal conductivity has the minimum value in the case of Iron metal in comparison with the other metals in this study. This makes the heat transfer in Iron slower than from the other metals under study. Figures (6-9) show the behavior of temperature distribution for the other metals under study (Copper and Aluminum).

Figures (6-9) show that the minimum center temperature found in Copper metal. This is because of the high thermal conductivity of Copper with respect to the other metals under study.

Figures (4-9) show that the maximum temperature for all metals under this study is located at the center of the solid sphere. The reason from that is the uniform heat generation which is distributed uniformly throughout the solid sphere. The uniformly generated heat makes the temperature having the maximum value in the center of the model under study.

\section{CONCLUSIONS}

Numerical and analytical study has been made for a model of a solid sphere. The study was taken into account the effect of heat generation. The comparison between the constant and variable thermal conductivity had been achieved. The comparison between the numerical and analytical study had been made and the percentage of the matching between the numerical and analytical study is very high. For a constant thermal conductivity the percentage error between the results is $0 \%$ which is mean a high accurate in the technique which is used in this study. In the case of the variable thermal conductivity the percentage error between the numerical and analytical study is $0.06 \%$. This is also means that the results that had been achieved in this technique are acceptable to a high degree. The results show also that the curvature of temperature distribution becomes important in a solid sphere model when the radius is more than $0.5 \mathrm{~m}$. Under this value the temperature distribution behavior takes a linear shape. The effect of the variable thermal conductivity becomes significant at a higher temperature of the surface (above 150 $\mathrm{C}^{\mathrm{o}}$ ). Iron having the most humping temperature distribution than the other metals under study (Copper and Aluminum). This is because of the low thermal conductivity of Iron in a comparison with the Copper and Aluminum.

\section{APPENDIX-A}

In this appendix, the derivation of the equation of temperature distribution will be achieved. In the case of the constant thermal conductivity, temperature distribution becomes:

$\frac{1}{\mathrm{r}^{2}} \frac{\partial}{\partial \mathrm{r}}\left(\mathrm{kr}^{2} \frac{\partial \mathrm{T}}{\partial \mathrm{r}}\right)+\dot{\mathrm{q}}=0$

$$
\begin{aligned}
& \mathrm{kr}^{2} \frac{\partial \mathrm{T}}{\partial \mathrm{r}}=-\dot{\mathrm{q}} \frac{\mathrm{r}^{3}}{3}+\mathrm{C}_{1} \rightarrow \frac{\partial \mathrm{T}}{\partial \mathrm{r}}=\frac{-\dot{\mathrm{q}} \mathrm{r}}{3 \mathrm{k}}+\frac{\mathrm{C}_{1}}{\mathrm{kr}^{2}} \\
& \mathrm{dT}=\frac{-\dot{\mathrm{q}} \mathrm{r}}{3 \mathrm{k}} \mathrm{dr}+\frac{\mathrm{C}_{1}}{\mathrm{kr}^{2}} \mathrm{dr} \rightarrow \mathrm{T}(\mathrm{r})=\frac{-\dot{\mathrm{q}} \mathrm{r}^{2}}{6 \mathrm{k}}-\frac{\mathrm{C}_{1}}{\mathrm{kr}}+\mathrm{C}_{2}
\end{aligned}
$$

Boundary Conditions:

$$
\begin{aligned}
& \text { At } r=0, \quad \frac{\partial T}{\partial r}=0 \quad, \text { at } r=R, \quad T=T_{s} \\
& \therefore \mathrm{C}_{1}=0, \quad C_{2}=T_{s}+\frac{\dot{\mathrm{q}} R^{2}}{6 k} \\
& \therefore \quad T(r)=T_{s}+\frac{\dot{\mathrm{q}} R^{2}}{6 k}\left(1-\frac{\mathrm{r}^{2}}{\mathrm{R}^{2}}\right)
\end{aligned}
$$

Equation (a3) represents the temperature distribution for a solid sphere subjected to a uniform heat generation with a constant thermal conductivity.

In the case of a variable thermal conductivity, the derivation of the equation of the temperature distribution is:

$\frac{1}{\mathrm{r}^{2}} \frac{\partial}{\partial \mathrm{r}}\left(\mathrm{kr}^{2} \frac{\partial \mathrm{T}}{\partial \mathrm{r}}\right)+\dot{\mathrm{q}}=0$

$\mathrm{k} \frac{\partial \mathrm{T}}{\partial \mathrm{r}}=\frac{-\dot{\mathrm{q}} \mathrm{r}}{3}+\frac{\mathrm{C}_{1}}{\mathrm{r}^{2}} \rightarrow\left(\mathrm{k}_{0}+\mathrm{k}_{0} \beta \mathrm{T}\right) \partial \mathrm{T}=\frac{-\dot{\mathrm{q}} \mathrm{r}}{3} \partial \mathrm{r}+\frac{\mathrm{C}_{1}}{\mathrm{r}^{2}} \partial \mathrm{r}$

$\mathrm{k}_{0} \mathrm{~T}+\frac{\mathrm{k}_{0} \beta}{2} \mathrm{~T}^{2}=\frac{-\dot{\mathrm{q}} \mathrm{r}^{2}}{6}-\frac{\mathrm{C}_{1}}{\mathrm{r}}+\mathrm{C}_{2}$

$\mathrm{T}^{2}+\frac{2}{\beta} \mathrm{T}+\frac{\dot{\mathrm{q}} \mathrm{r}^{2}}{3 \mathrm{k}_{0} \beta}+\frac{2 \mathrm{C}_{1}}{\mathrm{rk}_{0} \beta}-\frac{2 \mathrm{C}_{2}}{\mathrm{k}_{0} \beta}=0$

Boundary conditions:

$$
\begin{aligned}
& \text { At } \mathrm{r}=0, \quad \frac{\partial \mathrm{T}}{\partial \mathrm{r}}=0 \quad \text { at } \mathrm{r}=\mathrm{R}, \quad \mathrm{T}=\mathrm{T}_{\mathrm{S}} \\
& \mathrm{C}_{1}=0, \quad \mathrm{C}_{2}=\mathrm{k}_{0} \mathrm{~T}_{\mathrm{S}}+\frac{\mathrm{k}_{0} \beta}{2} \mathrm{~T}_{\mathrm{S}}^{2}+\frac{\dot{\mathrm{q}} \mathrm{R}^{2}}{6} \\
& \mathrm{~T}^{2}+\frac{2}{\beta} \mathrm{T}+\frac{\dot{\mathrm{q}} \mathrm{r}^{2}}{3 \mathrm{k}_{0} \beta}-\frac{2 \mathrm{C}_{2}}{\mathrm{k}_{0} \beta}=0 \\
& \mathrm{~T}(\mathrm{r})=\frac{-\frac{1}{\beta} \pm \sqrt{\left(\frac{2}{\beta}\right)^{2}-4\left(\frac{\dot{\mathrm{q}} \mathrm{r}^{2}}{3 \mathrm{k}_{0} \beta}-\frac{2 \mathrm{C}_{2}}{\mathrm{k}_{0} \beta}\right)}}{2}
\end{aligned}
$$

By substitute the value of $\mathrm{C}_{2}$ in equation (a4) and rearranging:

$\mathrm{T}(\mathrm{r})=-\frac{1}{\beta} \pm \sqrt{\left(\frac{1}{\beta}+\mathrm{T}_{\mathrm{S}}\right)^{2}+\frac{\dot{\mathrm{q}} \mathrm{R}^{2}}{3 \mathrm{k}_{0} \beta}\left(1-\frac{\mathrm{r}^{2}}{\mathrm{R}^{2}}\right)}$

Equation (a5) represents the temperature distribution of a solid sphere subjected to a uniform heat generation with a variable thermal conductivity.

$\frac{\partial}{\partial \mathrm{r}}\left(\mathrm{kr}^{2} \frac{\partial \mathrm{T}}{\partial \mathrm{r}}\right)=-\dot{\mathrm{q}} \mathrm{r}^{2} \rightarrow \partial\left(\mathrm{kr}^{2} \frac{\partial \mathrm{T}}{\partial \mathrm{r}}\right)=-\dot{\mathrm{q}} \mathrm{r}^{2} \partial \mathrm{r}$ 


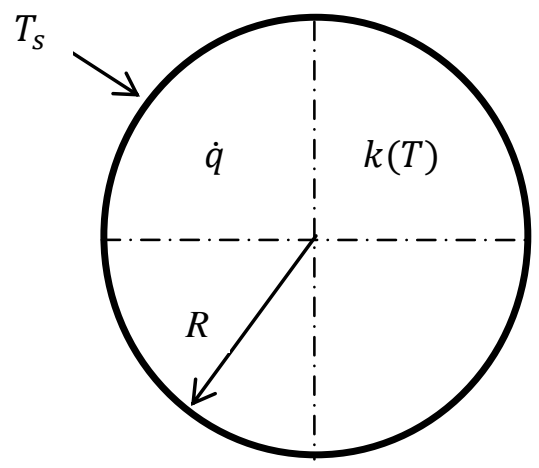

Fig 1: Profile of a solid sphere with a certain surface temperature.
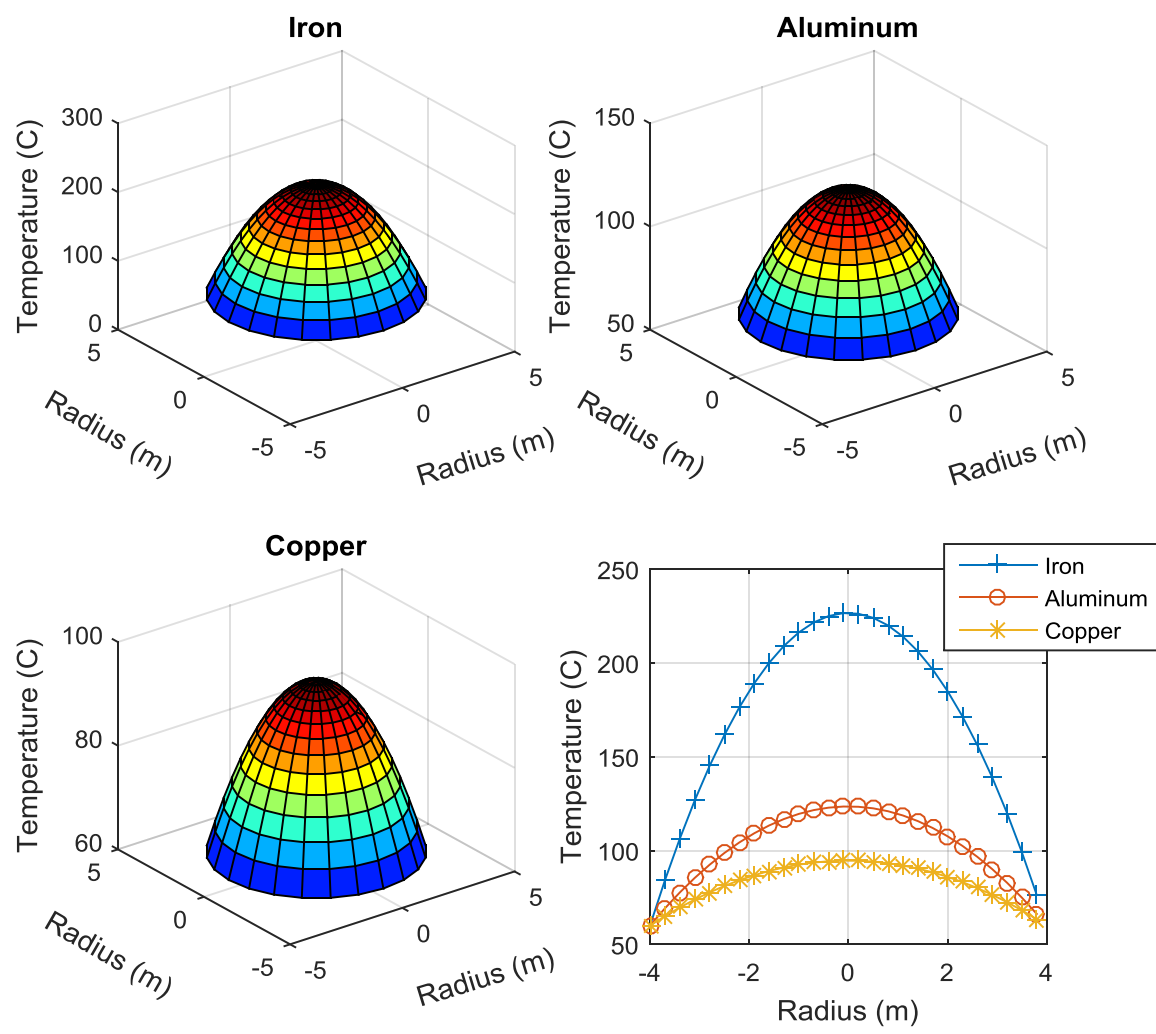

Fig 2: Temperature distribution of a solid sphere with constant thermal conductivity. 
International Journal of Computer Applications (0975 - 8887)

Volume 168 - No.2, June 2017
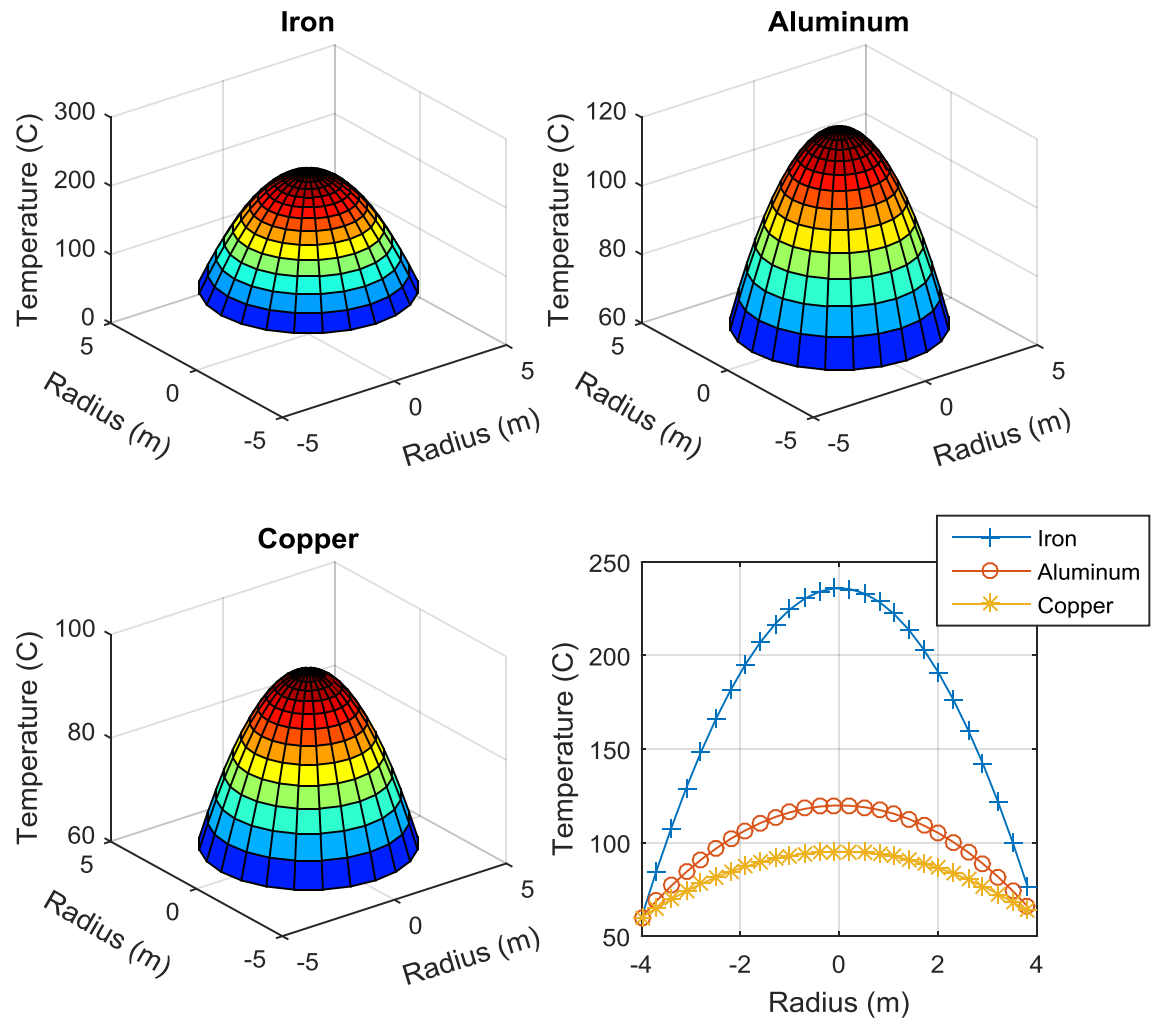

Fig 3: Temperature distribution of a solid sphere with variable thermal conductivity.

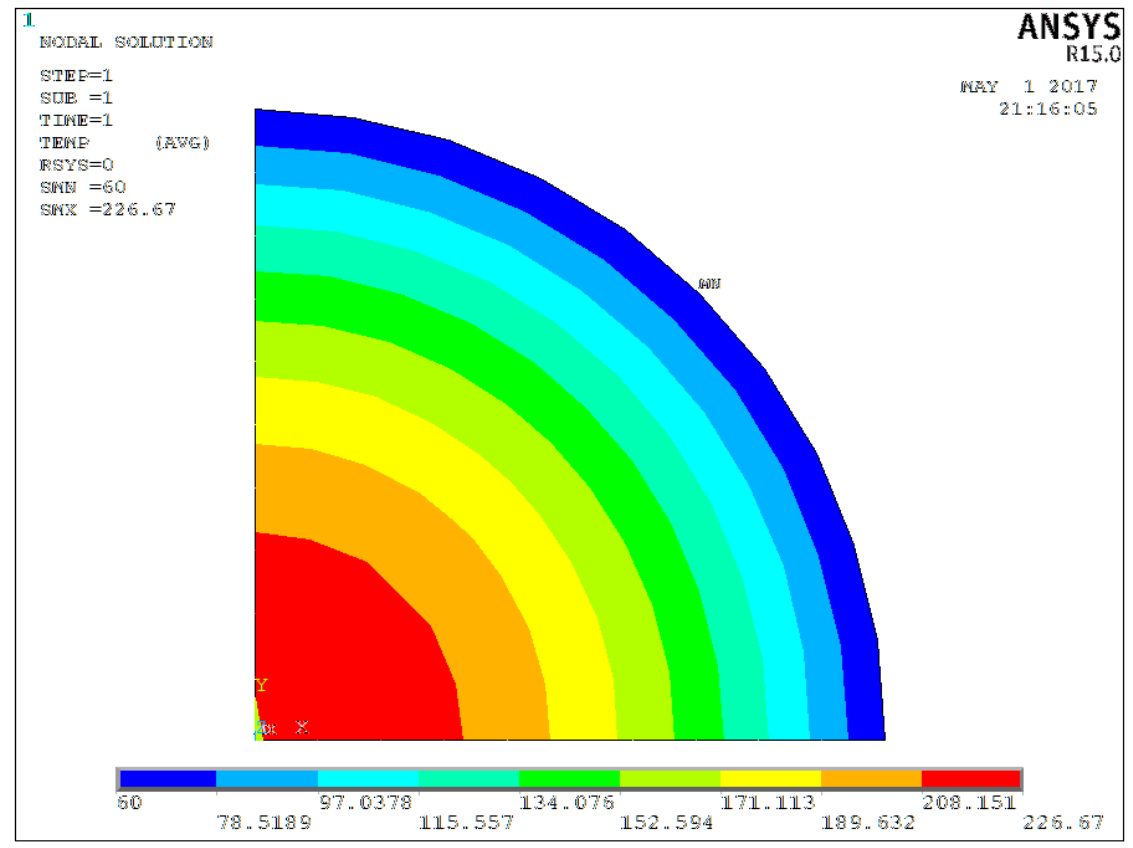

Fig 4: Temperature distribution of a solid sphere with constant thermal conductivity (Iron model).

34 


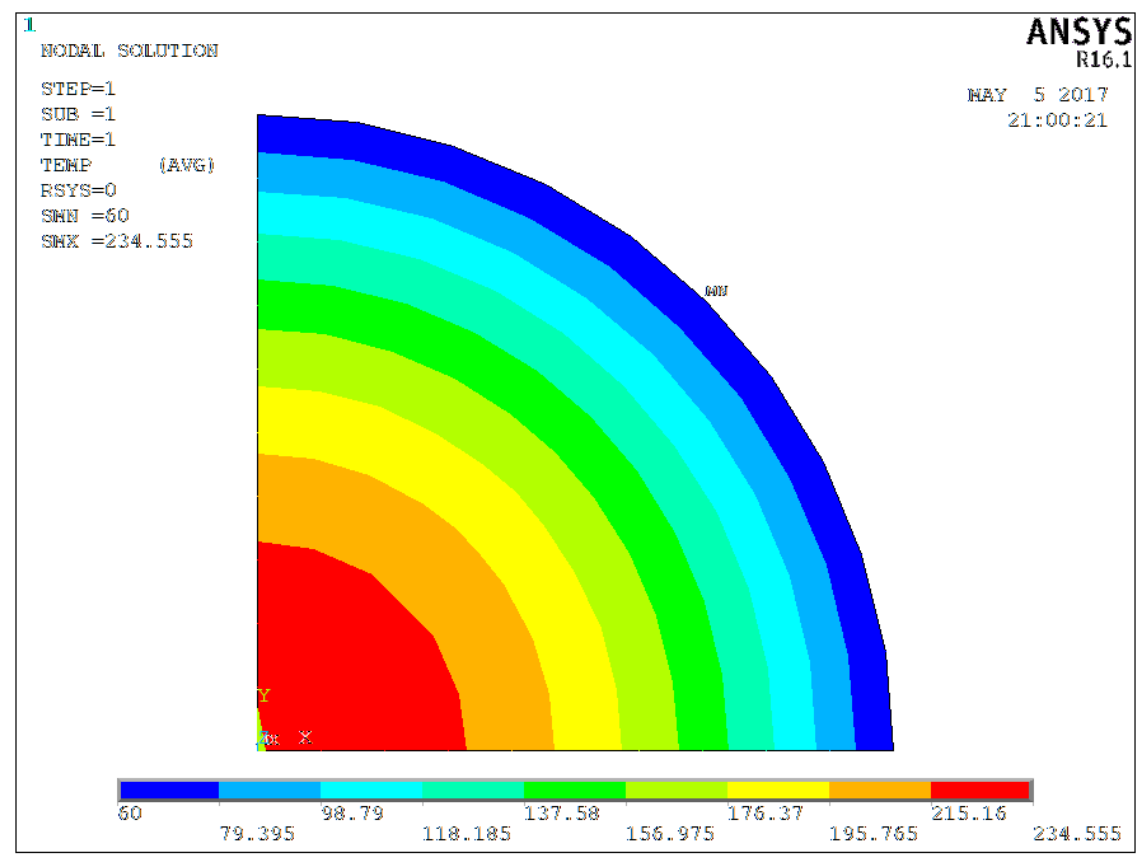

Fig 5: Temperature distribution of a solid sphere with variable thermal conductivity (Iron model).

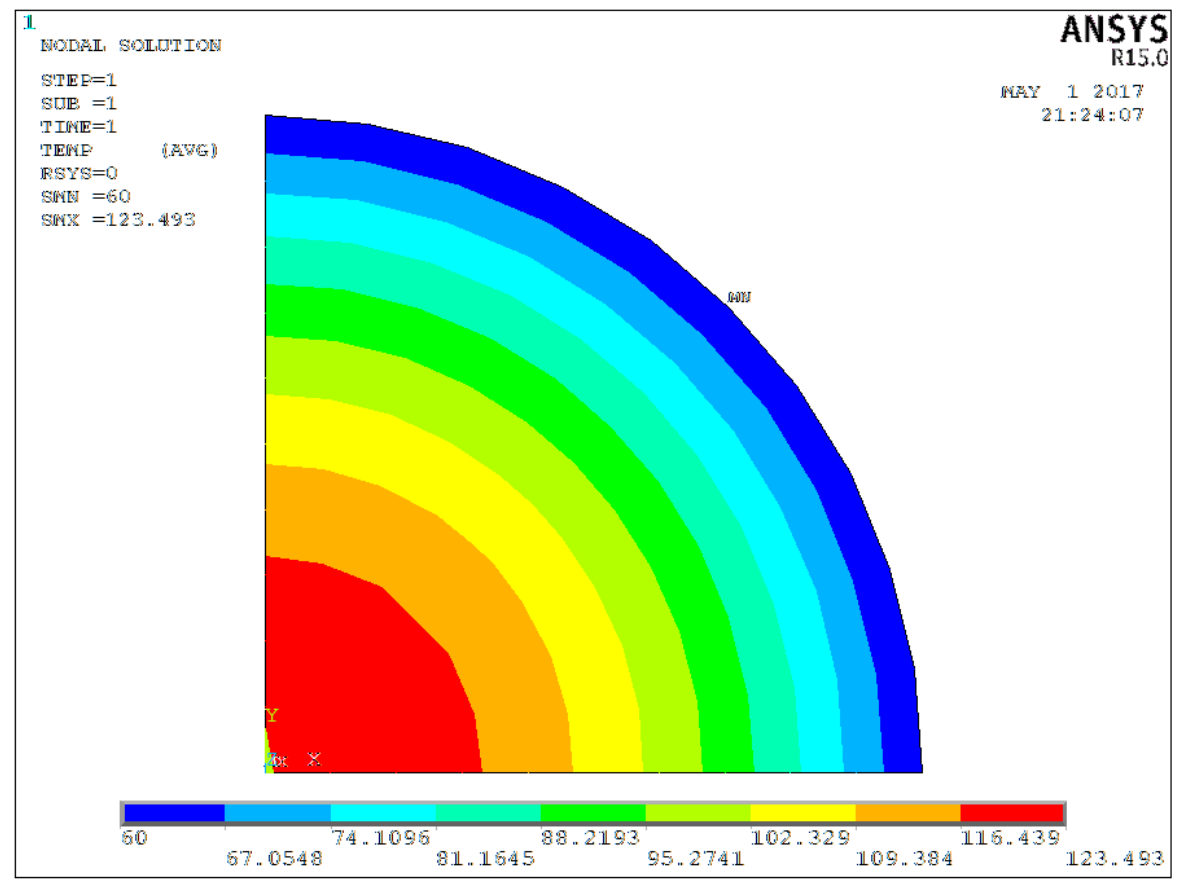

Fig 6: Temperature distribution of a solid sphere with constant thermal conductivity (Aluminum model). 


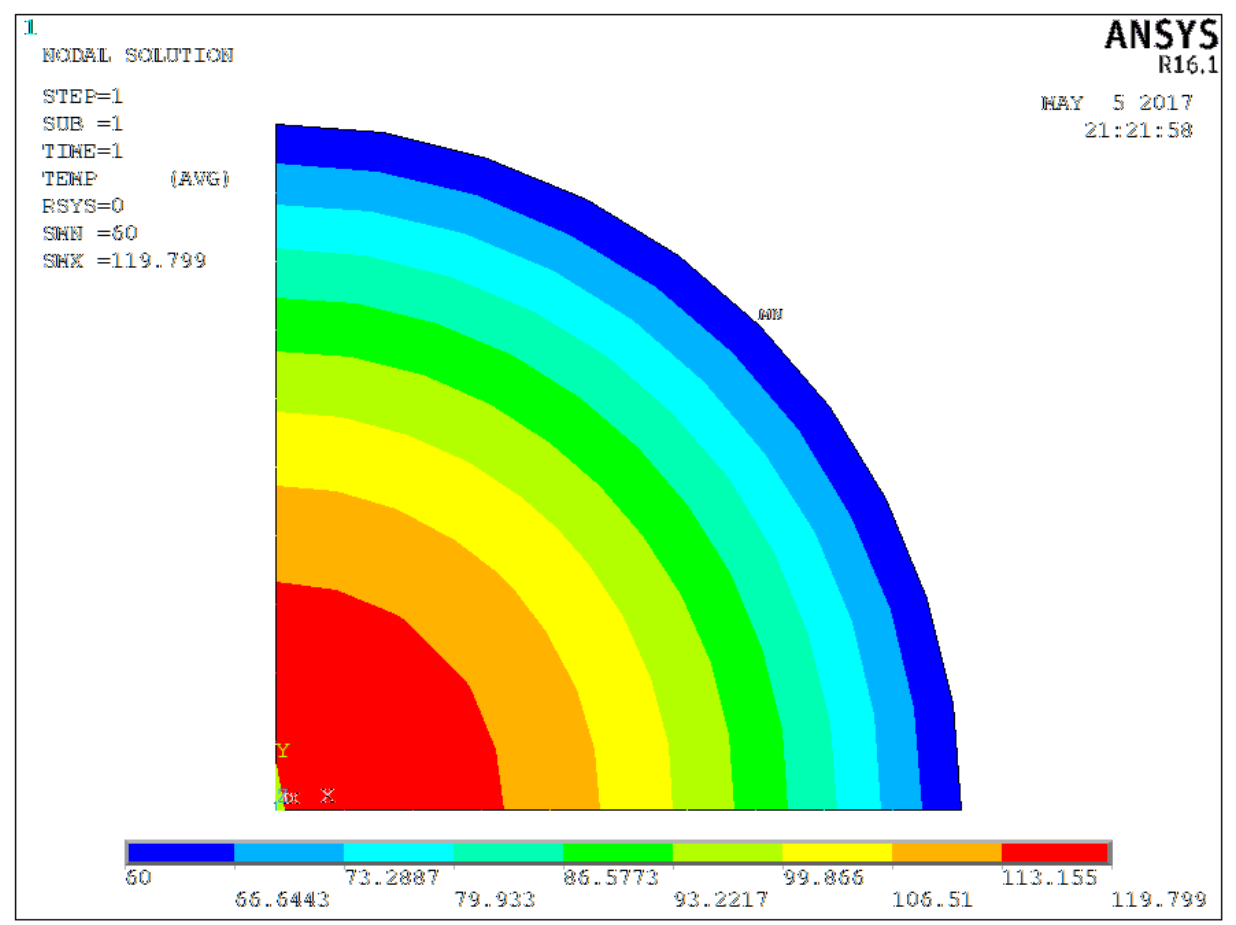

Fig 7: Temperature distribution of a solid sphere with variable thermal conductivity (Aluminum model).

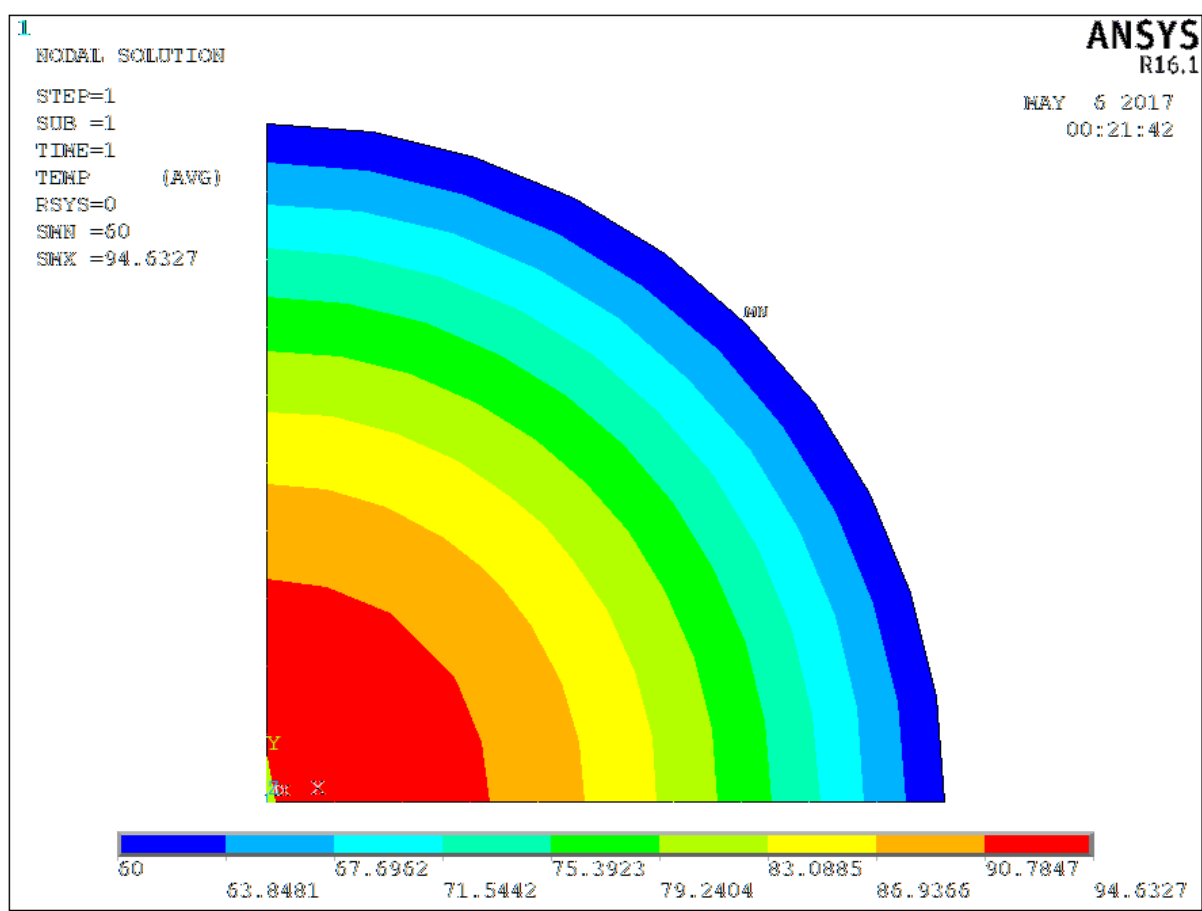

Fig 8: Temperature distribution of a solid sphere with constant thermal conductivity (Copper model). 


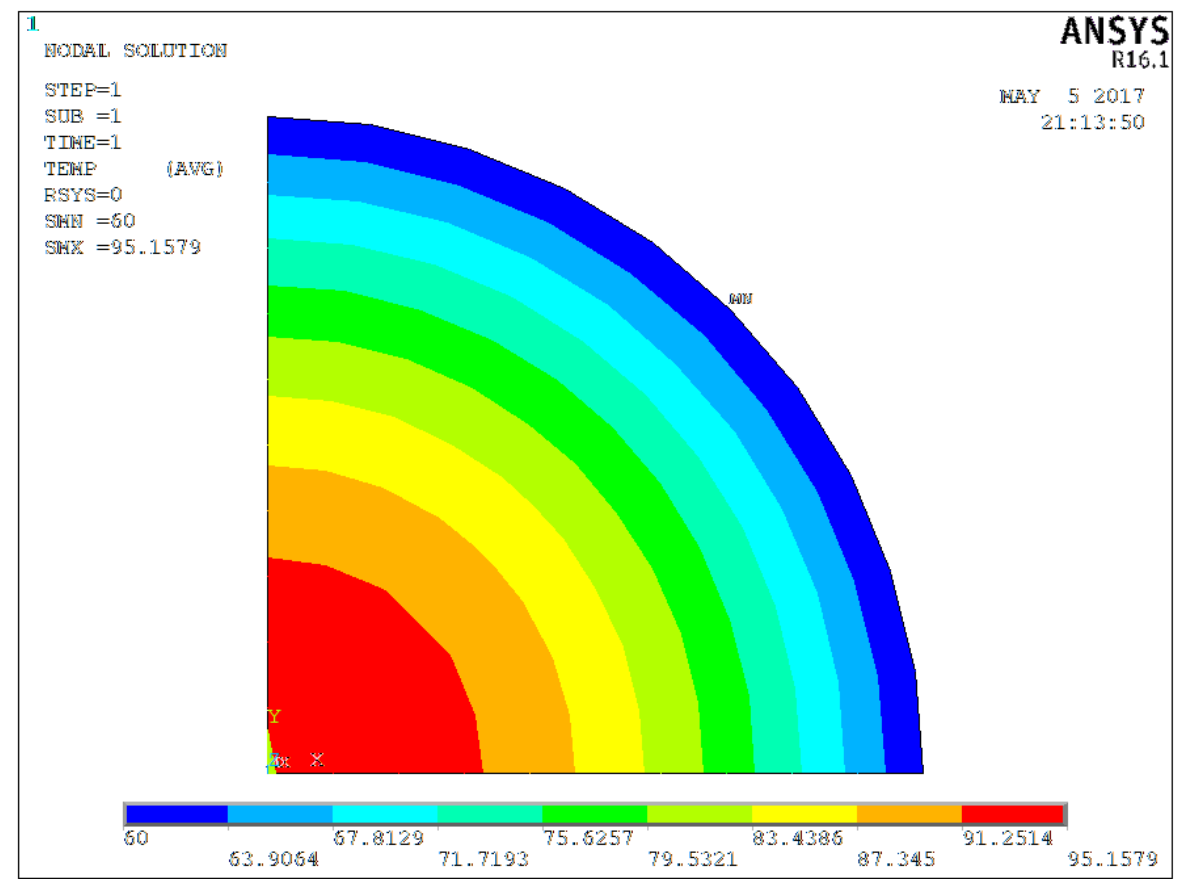

Fig 9: Temperature distribution of a solid sphere with variable thermal conductivity (Copper model).

\section{REFERENCES}

[1] Kuen-Tzong Lu and Hong-Sen Kou, The Effective Thermal Conductivity of Porous Material With Spherical Inclusion in a Tetragonal or Simple Cubic Array, Int. Comm. Heat Mass Transfer, Vol. 20, pp. 489-500, 1993.

[2] H. C. Unal, Temperature distribution in a plate with temperature-dependent thermal conductivity and internal heat generation, Int..I. Heat Mass Transfer. Vol.32, No. 10, pp. 1917-1926, 1989.

[3] Jaroslav PFANN, Determination of the Steady Temperature Distribution in Solids with Internal Heat Sources and Temperature-Dependent Thermal Conductivity.

[4] Hussein K. Jobair, Analytical Study of Temperature Distribution of a Plane Wall Subjected to a Constant Heat Generation with Variable Thermal Conductivity Using Different Materials, International Journal of Science and Research, Volume 6 Issue 3, pp. 882-888, March 2017.
[5] Davood Domiri GANJI, Zaman Ziabkhsh GANJI, and Hosain Domiri GANJI, Determination of Temperature Distribution for Annular Fins With Temperature Dependent Thermal Conductivity by (HPM), THERMAL SCIENCE, Year 2011, Vol. 15, Suppl. 1, pp. S111-S115.

[6] En-Bo Wei , Jin-Bao Song , Ji-Wei Tian, Thermal conductivity of graded spherical composites with contact resistance, Physics Letters A 319 (2003) 401-405.

[7] G. C. Bussolino, J. Spigiak, F. Righini, A Rosso, P. C. Cresto, and R. B. Roberts, Evaluation of Thermal Conductivity from Temperature Profiles, International Journal of Thermophysics, Vol. 14, No. 3, 1993.

[8] Theodore L. Bergman, Adrienne S. Lavine, Frank P. Incropera, David P. Dewitt, Fundamental of Heat and Mass Transfer, Seventh Edition. 